\title{
GHB: Un análisis
}

\author{
Louisa Degenhardt
}

National Drug and Alcohol Research Centre. University of New South Wales. Sydney. Australia.

Enviar correspondencia: L. Degenhardt. University of New South Wales. Sydney. New South Wales 2052. AUSTRALIA

Ph: 6129385 0230. Fax: 6129385 0222. Email: I.degenhardt@unsw.edu.au.

\section{Resumen}

Se revisa la naturaleza, efectos y daños asociados al GHB (gamma-hidroxibutirato), una droga con efectos depresores sobre el sistema nervioso central y se resumen las investigaciones realizadas para analizar los efectos sobre consumidores recreativos. El consumo ilegal de GHB se ha extendido, en la última década, en ciertos países desarrollados, especialmente entre consumidores de otras drogas ilegales. Las personas que utilizan GHB suelen consumir también otras drogas, ingiriendo ambas de una manera combinada, lo que puede incrementar los daños asociados. La existencia de un importante número de informes sobre casos de sobredosis de GHB y el elevado número de casos de sobredosis referidos por los propios consumidores, indican que el riesgo de sobredosis con esta droga es muy alto. La mayoría de las sobredosis no tienen consecuencias fatales y muchas finalizan con una total recuperación, pero también se han indicado algunas muertes, además de los posibles daños que pueden aparecer mientras los consumidores se encuentran inconscientes. El consumo regular de GHB genera dependencia y ésta es común en consumidores habituales de larga duración. El tratamiento de la dependencia generada por GHB se encuentra aún en fase de desarrollo, pero se han descrito tratamientos para el síndrome de abstinencia, lo que parece relevante dada la gravedad de dicho síndrome.

Palabras claves: Gamma-hidroxibutirato (GHB), consumo recreativo, efectos adversos, sobredosis, dependencia.

\section{Summary}

This paper gives an overview of the nature, effects and related harms of gamma-hydroxybutyrate (GHB), a drug with depressant effects upon the central nervous system. It also gives a summary of research conducted to date on those who use GHB for recreational purposes. The illicit use of GHB has increased in a number of developed countries over the past decade, largely among users of other illicit drugs. Those using illicit GHB seem to be users of other illicit drugs, who tend to use GHB in combination with other drugs, which may increase the harms associated with such use. Given numerous reports of overdose on $\mathrm{GHB}$, and high rates of self-reported overdose on GHB, it would appear that the risk of overdose on this drug is high. The majority of overdoses reported are non-fatal, and many remit with full recovery, but deaths have been reported and there are a number of possible harms that may occur to users during unconsciousness. Dependence following regular use has been reported and appears to be common among heavy long-term users. Treatments for dependent GHB use remain to be developed, but treatment for withdrawal has been reported and seems important given the seriousness of withdrawal from GHB and related drugs.

Key Words: Gamma-hydroxybutyrate (GHB), recreational use, side effects, overdose, dependence. 


\section{INTRODUCCIÓN}

E Gamma-hidroxibutirato (GHB) es un ácido graso que aparece de manera natural en cuerpo humano $(1,2)$. Se sintetizó por primera vez en 1960 (1). Presenta algunas semejanzas con el neurotransmisor GABA, cuyos mecanismos específicos de acción están aún poco claros; parece que el GHB es un metabolito de GABA $(3,4)$. Una serie de indicios derivados de la investigación realizada señalan que puede ser un neurotransmisor: se han identificado zonas del cerebro de rata que constituyen lugares de unión de alta afinidad; el GHB cuenta con una enzima específica para su síntesis; es sintetizado en neuronas y existen mecanismos de recaptación química en las terminales presinápticas (3). El GHB se encuentra en el cerebro y se cree que se concentra en el hipotálamo y los ganglios basales (5).

Las primeras investigaciones evidenciaron que el GHB producía sedación y anestesia, indicando que se trataba de un depresor del sistema nervioso central (SNC), con algunas semejanzas con otros depresores tales como los benzodiacepinas y el alcohol $(6,7)$. Se cree que el GHB modula una serie de neurotransmisores tales como la dopamina, la serotonina y los opiáceos. (8-11). Estudios de discriminación de drogas han revelado que puede existir una tolerancia cruzada entre el GHB y el alcohol, aunque algunos han planteado una relación más compleja entre ambos (4). Al caracterizar el GHB, algunos estudios han llegado a la conclusión que los efectos de estímulo discriminatorio son muy similares a los del alcohol $(1,12,13)$. Existen indicios suficientes para pensar que el GHB intensifica los efectos depresores del sistema nervioso central tanto del alcohol como de otras drogas hipnótico/sedantes, y puede también incrementar los efectos de los opiáceos $(1,12)$.

Una vez ingerido, el organismo absorbe el $\mathrm{GHB}$, comenzando sus efectos una vez pasados 15 o 30 minutos, con un período de máximos efectos entre los 25 y 45 minutos después de la toma. (8). Los efectos parecen depender mucho de la dosis (6), llegando a aumentar espectacularmente los efectos con pequeños incrementos en las dosis ingeridas.

\section{UTILIZACIÓN DEL GHB CON FINES CLÍNI- $\cos$}

Las primeras investigaciones y los primeros consumos de GHB señalaron que esta droga podía consumirse de una manera segura (14) y sin que aparecieran efectos secundarios serios o se evidenciara abuso, habiéndose documentado su utilización durante cierto tiempo en Europa (7).

El GHB se ha investigado y utilizado para distintos usos clínicos. De acuerdo a su acción de naturaleza sedativa, el GHB se ha utilizado como una ayuda para dormir en personas con problemas de sueño, en particular con narcolepsia (15-17). Así, se pensó que la capacidad del GHB para inducir sueño nocturno en personas con narcolepsia, a través de la potenciación de los patrones "normales" de sueño, aconsejaba su utilización en esta enfermedad (1). El GHB ha resultado ser seguro y efectivo en el tratamiento de la narcolepsia en ensayos clínicos $(15,18)$, y en EEUU esta droga se encuentra aprobada por el United States FDA para su investigación con estos fines.

También se investigó el uso de el GHB como anestésico $(1,7)$. En 1964, el GHB se introdujo en Europa como agente anestésico, siendo utilizado sobre todo en niños $(19,20)$; sin embargo, su uso no se extendió al existir algunos casos de vómitos y convulsiones (21).

Un temprano estudio japonés realizado en seis adultos jóvenes varones encontró que el GHB estimulaba la liberación de hormona de crecimiento por parte de la hipófisis, con informes que indicaban incrementos de 9 a 16 veces en la hormona del crecimiento a los 30 a 60 minutos de la administración intravenosa del GHB (22). Probablemente este es uno de los principales factores que sirvieron 
de base al uso del GHB a lo largo de los ochenta y los noventa como un promotor del crecimiento y como facilitador de la pérdida de peso entre algunos grupos, tales como los culturistas $(17,23)$. A pesar de la evidencia del incremento, a corto plazo, de la hormona de crecimiento, no existe, sin embargo, ninguna evidencia que indique un incremento de la masa muscular a largo plazo.

Existen investigaciones que han analizado la efectividad del GHB como tratamiento ante la dependencia del alcohol y la desintoxicación de opiáceos (1, 7, 12, 24-26). Algunas investigaciones recientes han encontrado que era tan efectivo como la naltrexona (otro tratamiento farmacológico validado para la dependencia del alcohol) para reducir la ansiedad (craving) ante el alcohol y mejor que la naltrexona a la hora de mejorar la abstinencia generada por el alcohol, a corto plazo (27).

\section{CONSUMO DEL GHB SIN FINES MÉDICOS}

Existe documentación del consumo de GHB como droga recreativa en los últimos años en diferentes países del mundo. En la mayor parte de los casos la atención sobre este consumo se ha producido a partir de la documentación de casos de individuos que llegaban a urgencias en estado de inconsciencia. En EEUU, se publicaron informes, en 1990, en el sistema de información Morbidity and Mortality Weekly Reports (28), sobre intoxicaciones agudas por GHB en zonas como San Francisco y California.

Como había ocurrido en EEUU, la evidencia del consumo de GHB en Australia surgió a partir de informes esporádicos sobre personas que llegaban a los servicios de urgencias después de una sobredosis de GHB, y de la información de la red de control de aplicación de la ley (29). Estos informes se complementaron con sistemas de seguimiento de drogas en Nueva Gales del Sur y Australia del Sur, los cuales observaron el incremento en el consumo del GHB entre usuarios habituales de éxtasis muestreados anualmente (30,
31). Informes sobre el consumo e intoxicaciones han sido, posteriormente, documentados en EEUU (29, 32, 33), el Reino Unido (34), Canada (35), y España (36). En el caso de España, un estudio de admisiones en urgencias hospitalarias relacionadas con drogas ilegales concluyó que el GHB aparecía como la segunda droga ilegal más comúnmente nombrada en estos casos, representando un 3\% del total de admisiones en urgencias (36).

El GHB se conoce, en argot, por un amplio número de nombres diferentes tales como "GBH", "grievous bodily harm", "cherry meth", "Georgia Home Boy", "fantasia" ("fantasy"), "éxtasis líquido" ("liquid ecstasy"), " "jabón" ("soap"), "scoop", " Líquido E" ("Liquid E") y "Líquido X" ("Liquid X"). En algunos casos, los diferentes nombres pueden hacer pensar a los propios consumidores que realmente no están tomando GHB (especialmente en el caso de denominaciones tales como éxtasis líquido).

El aumento de las restricciones del uso de GHB en muchos países del mundo (ej. (1)) ha reducido el suministro de GHB legal. En los últimos años, la posibilidad para que los consumidores recreativos consigan GHB de manera ilegal se ha limitado de una manera importante.

Probablemente, como consecuencia de estas limitaciones se ha producido un incremento de informes sobre el consumo de 1,4butanediol (1,4-B) o gamma-butirolactona (GBL) (37). Estas son sustancias químicas similares al GHB que también aparecen de manera natural en el cuerpo (1), y que son metabolizadas como GHB en el cuerpo. Pueden ser consumidas como sustitutas del $\mathrm{GHB}$, pero son diferentes desde el punto de vista farmacológico. Los efectos de las dosis de GBL pueden ser también superiores que los de dosis equivalentes de GHB, lo cual podría llevar a problemas en relación con la valoración de la dosis (1).

Actualmente, existen evidencias para indicar que algunas personas producen GHB del $\mathrm{GBL}$, el cual (aunque limitado en algunos países) se puede obtener todavía como agente químico. En Internet se han podido conseguir 
"recetas" para llevar a cabo este proceso. Dadas las dificultades que rodean a la adecuada valoración de dosis de GHB y los potenciales efectos negativos si se ingiere demasiado GHB, la producción de esta droga por, en algunos casos, químicos sin experiencia, puede llevar a daños para aquellos consumidores que ingieran la sustancia.

La investigación en consumidores recreativos de GHB es muy escasa, así que, aunque existen conocimientos sobre los efectos y riesgos del consumo de GHB a partir de estudios clínicos, no se conocen bien los patrones de consumo y daños asociados al consumo recreativo de esta droga. En este tipo de consumos, factores adicionales como la fabricación ilegal del GHB (y, por lo tanto, la potencia variable), la diferente respuesta individual al GHB, las condiciones ambientales y el consumo de varias drogas al mismo tiempo, pueden incrementar los riesgos del consumo del GHB, tal como ocurre con todas las drogas ilegales; sin embargo, dado que la dosis puede ser un factor importante en el aumento de los efectos del GHB, puede ser que éste sea el aspecto más crucial en el consumo recreativo (ilegal) del GHB (1).

Para la realización de esta revisión, se identificaron dos estudios que se centraban en consumidores recreativos de GHB. Un estudio sobre 42 consumidores recreativos de GHB en Los Ángeles, California, encontró que éstos eran en su mayor parte jóvenes (en torno a la veintena), varones de raza blanca (38). Los consumidores informaron que utilizaban esta droga para aumentar sentimientos de euforia, sexualidad y relajación.

En el estudio australiano, la muestra seleccionada de 76 consumidores recreativos de GHB constituía un grupo con adecuado nivel educacional y con buen funcionamiento, que sólo recientemente se había involucrado en el consumo de GHB (39). Aunque es posible que estas trayectorias de ciclo corto de la muestra de consumidores de GHB pudieran reflejar una elevada tasa de discontinuidad entre las personas que utilizan GHB, también apoya la evidencia de que el consumo de GHB sólo se ha extendido como droga recre- ativa, en Australia, recientemente (29). También es coherente con la información de que el consumo de GHB con fines recreativos en países como EEUU y el Reino Unido es un fenómeno reciente (1). En este estudio, los consumidores de GHB habían tenido una amplia experiencia con otras drogas y, particularmente, un gran consumo reciente la lo largo de los últimos seis meses) de una variada gama de sustancias anfetamínicas y otras drogas habitualmente utilizadas por consumidores de party drug o club drug (40). Estas personas indicaron consumir GHB por razones similares a los individuos del estudio americano: para relajarse, por sus efectos euforizantes y porque aumentaba la sensación de sexualidad.

\section{PATRONES DE CONSUMO DE GHB}

En el estudio de Degenhardt et al. (39), muchos consumidores de GHB habían empezado a utilizar esta droga sólo recientemente (en el último año o dos años) y muchos no lo habían consumido de una manera extensiva (la mediana del número de veces consumida era 15).

El contexto típico de consumo de GHB era combinarlo con una gran variedad de otras drogas (solo un $5 \%$ informó que utilizaban GHB como única droga). Merece destacarse el hecho de que tres de cada diez consumidores de GHB informaron que bebían más de cinco bebidas alcohólicas cuando consumían $\mathrm{GHB}$, y 2 de cada 10 informaran que consumían ketamina al mismo tiempo que GHB. Dados los efectos depresivos y disociativos de estas sustancias, su consumo con GHB puede estar situandoo a los consumidores ante un mayor riesgo de efectos adversos, particularmente si quedan inconscientes; lo que puede incrementar los riesgos de los consumidores ante accidentes o heridas.

En el estudio americano, los patrones de consumo fueron mucho más intensos: casi la mitad informó que consumían GHB dos o más días por semana (38). A menudo lo con- 
sumían dos o tres veces en una ocasión de uso y tomaban 1-3 cápsulas.

\section{EFECTOS COLATERALES DEL CONSUMO DE GHB}

Los efectos colaterales del GHB han sido documentados tanto en investigación clínica, como en un pequeño número de estudios en consumidores recreativos. La investigación clínica y los casos de estudio han demostrado que los efectos adversos del GHB incluyen mareos, náuseas, debilidad, confusión y agitación, somnolencia y coma $(1,6,17,41)$. Los estudios clínicos y el uso inicial del GHB como anestético indicaron que también puede inducir actividad convulsiva (41), aunque esta posibilidad no ha sido directamente substanciada utilizando evaluación con EEG. Se ha informado sobre efectos adversos con dosis entre 2 y 30 gramos de polvo de GHB (17).

En el estudio americano de consumidores de GHB, más de la mitad de la muestra informó sobre un aumento de sudoración y pérdida de conciencia; entre un cuarto y la mitad informó de náuseas, alucinaciones, dolores de cabeza y vómitos. Una minoría informó que experimentaba convulsiones cuando consumía GHB (38).

En el estudio australiano de consumidores de GHB lo que resultó más notable fue que, a pesar del poco tiempo que estos consumidores recreativos llevaban consumiendo GHB y del hecho de que la mayoría de los consumidores hubieran tenido una experiencia limitada con la droga, los informes de efectos adversos fueron frecuentes en este grupo: el número medio de efectos secundarios experimentado fue de 6,5 (de un total posible de 14) y el $99 \%$ informó de, al menos, un efecto colateral (39). Estos efectos secundarios incluyeron vómitos, sudoración profusa, mareos, confusión y convulsiones. Mientras que muchos usuarios pensaron que estos efectos se debían al consumo de GHB, los patrones de uso múltiple de drogas de este grupo hacen pensar que es difícil estar seguro de que drogas (o combinación de drogas) es responsable de los mismos. Sin embargo, hay que destacar que estos efectos colaterales son similares al tipo de efectos negativos que se han observado en estudios clínicos de consumo de GHB (1). Estos resultados sugieren que incluso entre consumidores recreativos recientes e infrecuentes se experimentan significativos efectos adversos por el GHB, que pueden poner a los usuarios en riesgo de sufrir daños.

\section{SOBREDOSIS POR GHB}

La mayoría de la investigación sobre sobredosis de GHB se ha realizado sobre la base de casos de intoxicación, sobredosis o muerte. Muchos de ellos se han basado en el análisis de un pequeño número de casos en los que se sospechaba que las personas habían experimentado una sobredosis de GHB (6, 37, 42-45); por otra parte, existen evidencias de una intoxicación colectiva de GHB por la que 31 participantes de un rave party en California en el Año Nuevo de 1996 tuvieron que ser hospitalizados (46). Se han realizado dos estudios que llevan a cabo análisis de todos los casos admitidos en urgencias, en Barcelona, España (36), y San Francisco, EEUU (47).

En el estudio español de admisiones en urgencias, los investigadores encontraron que el caso típico de sobredosis de GHB era un varón, en la veintena, durante un fin de semana y muy a menudo habiendo consumido también alcohol (73\%) y otras drogas ilegales $(85 \%)$ y en estado de inconsciencia (36). No hubo muertes entre estos casos y siempre se produjo la recuperación.

En el estudio de San Francisco los resultados fueron similares: la mayoría de casos eran varones con una edad media de 28 años (47). Las tasas de consumo de otras drogas eran menores, con un 39\% de casos incluyendo alcohol y un $28 \%$ incluyendo otras drogas ilegales. Temperatura corporal baja, bradi- 
cardia e inconsciencia eran también rasgos comunes a estos casos.

Además de documentar la existencia de sobredosis asociadas a GHB, lo que resulta también de interés son las tendencias en el número de sobredosis relacionadas con GHB. Probablemente los mejores datos sobre este aspecto vienen de la Red Americana de Aviso sobre Abuso de Drogas (DAWN), cuyo sistema de datos ha mostrado incrementos dramáticos en el número de casos en urgencias de personas que habían sufrido sobredosis de GHB o habían tenido problemas relacionados con esta droga en EEUU. El numero de personas que se presentaron en urgencias por sobredosis de GHB o por problemas relacionados con el GHB aumentó desde 20 en el año 1992 a casi 5.000 en el $2000(33,48)$. También se han recibido informes de muertes por sobredosis relacionadas con GHB en EEUU (49), la mayoría de las cuales incluían también consumo de otras drogas $(49,50)$, aunque hay un caso publicado atribuido exclusivamente al consumo de GHB (51). En el estudio español se produjo un aumentó significativo en la proporción de casos de sobredosis por GHB entre 1989 y 2000-2001 (36). Dada esta evidencia y la mayor atención que se presta al GHB en los últimos años, parece que las sobredosis relacionadas con GHB han aumentado en varios países.

Existen pocos datos sobre el riesgo de sobredosis del GHB entre consumidores recreativos. En el estudio americano de GHB, más o menos la mitad confesó haber perdido la conciencia, mientras que $15-30 \%$ informó de "sobredosis" después de consumir GHB (38).

En el estudio australiano, la mitad (53\%) de la muestra de consumidores de GHB había sufrido sobredosis en algún momento (sobredosis se definió como pérdida de conciencia e incapacidad para estar despierto) (52). De aquellos que habían consumido GHB más de 15 veces, un $75 \%$ había tenido sobredosis al menos una vez; un tercio (33\%) de los que habían tenido sobredosis, la habían sufrido más de tres veces; una persona informó haber padecido 100 sobredosis; dos tercios (63\%) vieron a otra persona sufrir una sobredosis después de consumir GHB (52).

Existían pocas diferencias entre los que sufrieron y no sufrieron sobredosis de GHB (52). No existían divergencias entre los dos grupos en lo que respecta a las tasas de consumo de drogas a lo largo del a vida, el consumo de otras drogas en los últimos seis meses o en lo que se refiere a patrones típicos de consumo de otras drogas cuando utilizaban GHB. Aparentemente, sí había diferencias significativas entre aquellos que habían padecido y los que no habían padecido sobredosis en términos de su experiencia con el consumo de GHB (tiempo de consumo, número de veces que la droga había sido consumida, frecuencia de consumo), y muchos de aquellos que confesaron sobredosis informaron que ésta había ocurrido únicamente en una ocasión especial, tal como la noche de fin de año, un cumpleaños o un gran baile (52). Casi todos (93\%) dijeron que otros habían estado presentes cuando ocurrió la sobredosis. Los lugares más comunes donde se produjeron casos de sobredosis fueron los siguientes: en casa de alguien $(40 \%)$, en una discoteca ( $25 \%)$, en un baile (20\%) o en un lugar público (10\%). Las razones más comunes que los consumidores refieren, así como, su percepción de la sobredosis de GHB, en general, se relaciona con la potencia del GHB, la frecuencia de las dosis y la cantidad consumida. Estos datos son coherentes con lo que se conoce sobre la curva dosis-respuesta de GHB, en la cual pequeños incrementos en la cantidad de GHB consumida pueden producir enormes diferencias en los efectos sobre el consumidor (1).

Se debe destacar que algunos de estos consumidores australianos no percibían la sobredosis como algo negativo y muchos confesaron que habían obtenido información vía Internet y que pensaban que la sobredosis de GHB no era peligrosa (52). Estas percepciones del riesgo deben ser consideradas en cualquier intervención que pretenda minimizar los daños asociados con GHB, esforzándose en educar a los consumidores sobre 
los riesgos que pueden sobrevenir después de quedar inconscientes (ej. caída, heridas, riesgo de ahogamiento particularmente si existen vómitos).

Un estudio en varones participantes en circuitos de fiestas en Estados Unidos (eventos de tres días de baile periódicos) indicó que un cuarto (25\%) de la muestra habían informado de un "consumo abusivo" en alguna de las fiestas a lo largo del último año (éste se definió como haberse desvanecido, haber requerido asistencia médica o no haber sido capaces de cuidarse de si mismo). Un 53\% de estos varones dijeron que la droga que se consumía más a menudo era el GHB, mientras que un $45 \%$ citaba ketamina y $\mathrm{GHB}$, y un $34 \%$ citaba éxtasis. Curiosamente, las tasas de consumo de GHB eran más elevadas en la situación de sobredosis que en la fiesta del anterior fin de semana (que era el $25 \%$ ), además, las tasas de consumo, mencionadas en el incidente de sobredosis eran más bajas para las otras drogas citadas en comparación con el fin de semana previo (el consumo de ketamina fue de $58 \%$, alcohol $58 \%$ y éxtasis 75\%) (53).

Se ha discutido que uno de los aspectos más peligrosos del consumo recreativo de GHB son las dificultades para determinar la potencia y la dosis correcta de $\operatorname{GHB}(6,17)$. Esto puede estar en relación con la sobredosis relacionada con GHB. Esta idea es coherente con los resultados del estudio australiano que indicaron que el consumo de GHB en si mismo era el único factor de riesgo distintivo en la sobredosis (52). También es coherente con el estudio del circuito de fiestas americano, donde tasas más elevadas de consumo de GHB y tasas más bajas de otros consumos diferenciaban, aparentemente, los incidentes de sobredosis de lo que pasaba en otros circuitos de fiestas donde éstos no ocurrían. (53).

El manejo de la sobredosis de GHB ha sido objeto de un cierto debate. En muchos casos se ha recomendado un enfoque conservador en su tratamiento $(54,55)$. En los casos más graves se puede necesitar la intubación y la ventilación (54). Algunos autores han reporta- do que para casos graves se puede también usar la fisostigmina (54). La fisostigmina se ha empleado como un agente neutralizador del GHB y puede ser de utilidad para pacientes muy afectados.

\section{DEPENDENCIA DEL GHB}

Existe alguna evidencia de que se puede desarrollar tolerancia y dependencia física con el uso de GHB, lo que viene sugerido por la existencia un síndrome de abstinencia que puede incluir insomnio, calambres musculares, temblores y ansiedad (6). Se han publicado casos de dependencia de GHB entre consumidores crónicos $(6,23,56,57)$. Estos han ocurrido después de periodos de consumo intensivo y regular de GHB.

En el estudio de Degenhardt et al, aproximadamente el $4 \%$ de los participantes fueron clasificados como dependientes del GHB (39): aunque esta frecuencia estimada es baja, indica que la dependencia del GHB parece tener lugar entre consumidores recreativos. Este ratio de frecuencia podría también aumentar con el paso del tiempo, dado que el GHB sólo se utiliza con fines recreativos en Australia desde tiempos recientes. Como apoyo a ésto, el estudio americano de consumidores de GHB que comprendía una muestra a más largo plazo y con mayores tasas de consumo, encontró que el $21 \%$ de la misma informaba haber creído que eran "dependientes" del GHB en algún momento (38).

También se han producido casos de abstinencia de GHB y de GBL tras un periodo intenso y regular de consumo $(56,58-61)$. Los síntomas referidos durante la abstinencia incluyeron: insomnio, irritabilidad, paranoia, alucinaciones auditivas y visuales, taquicardia ligera, hipertensión, náuseas y vómitos. Los síntomas de abstinencia comenzaron generalmente a las pocas horas de abandonar el consumo, apareciendo, posteriormente, síntomas graves si no se producía un ingreso inmediato para una desintoxicación médica. 
Los tratamientos descritos para abordar el síndrome de abstinencia incluyen el uso de anticonvulsivantes para reducir la agitación y el riesgo de convulsiones (56). En los casos más graves de abstinencia se ha reportado el éxito del uso de barbitúricos, de dosis muy elevadas de benzodiacepinas de acción corta, de estabilizadores del ánimo y de antipsicóti$\cos (56,58,60,61)$. Un estudio refiere que las benzodiacepinas eran el único tratamiento efectivo en algunos casos (60). La aplicación temprana de estos tratamientos farmacológicos resultaba aparentemente beneficiosa para reducir la gravedad y la duración de los síntomas (56). Para aquellos con abstinencia a más largo plazo se requirió un seguimiento continuo, particularmente cuando existía gran agitación, delirio y psicosis (56). En un estudio, la abstinencia fue tan grave que acabó con la muerte del paciente (60).

\section{USO DE GHB EN CASOS SOSPECHOSOS DE ASALTO SEXUAL}

El consumo de GHB en casos de asalto sexual ha recibido alguna atención por parte de los medios de comunicación en los últimos años. Los casos de drink spiking y date rape en los que puede haber presencia de GHB parecen haberse incrementado en los últimos años, aunque hay problemas sobre la identificación y demostración del GHB en tales casos. Esto es debido a que el GHB se puede detectar en el organismo con independencia de que haya sido o no consumido; a que tiene una vida media corta en el organismo, a que es necesario realizar tests específicos y a la baja probabilidad de que se puedan tomar muestras en el periodo de tiempo requerido para detectar GHB. Aunque es un tema preocupante, se ha argumentado que las drogas más relevantes en los casos de asalto sexual facilitado por drogas son el alcohol, las benzodiacepinas y el cannabis (62).

\section{CONCLUSIONES}

El consumo de GHB parece haber aumentado en un cierto número de países desarrollados a lo largo de la última década. Los datos disponibles indican que los consumidores de GHB lo son también de otras drogas ilegales, tendiendo a ingerirlas de una manera combinada, lo que puede incrementar los daños asociados. Tras la limitación en la disponibilidad de GHB, se ha informado sobre la producción de GHB a partir de su precursor, el GBL, así como del consumo de precursores químicos y sustancias similares, GBL y 1,4-B. La existencia de numerosos casos publicados de sobredosis por GHB, así como las altas tasas de sobredosis manifestadas por los propios consumidores, llevan a hacer pensar que el riesgo de sobredosis con esta droga es elevado; la mayoría de los casos de sobredosis contabilizados no son fatales y generalmente acaban con una completa recuperación; sin embargo, se han producido muertes y existe un cierto número de posibles daños que pueden ocurrir a los consumidores de esta droga durante los periodos de inconsciencia. Se ha demostrado la dependencia después de un consumo regular y parece ser común entre los consumidores intensivos y a largo plazo. Los tratamientos para la dependencia de GHB están aun por desarrollar, pero ya se han descrito tratamientos para el síndrome de abstinencia, hecho que se considera significativo dada la gravedad del síndrome de abstinencia de GHB y de otras drogas similares.

\section{REFERENCIAS}

1. Nicholson, K. \& Balster, R. (2001) GHB: A new and novel drug of abuse, Drug and Alcohol Dependence, 63, 1-22.

2. Bessman, S. \& Fishbein, W. (1963) Gamma hydroxybutyrate - a new metabolite in brains, Federal Proceedings, 22, 334-342.

3. Colombo, C., Agabio, R., Lobina, C., Reali, R. \& Gessa, G. L. (1998) Involvement of GABAa and 
GABAb receptors in the mediation of discriminative stimulus effects of gammahydroxybutyric acid, Physiology and Behavior, 64, 293302.

4. Metcalf, B., Stahl, J., Allen, J., Woolfok, D. \& Soto, P. (2001) Discrimination of gammahydroxybutyrate and ethanol administered separately and as a mixture in rats, Pharmacology, Biochemistry and Behavior, 70, 31-41.

5. Snead, O. \& Morely, B. (1981) Ontogeny of gamma-hydroxybutyric acid. Regional concentration in developing rat, monkey and human brain, Brain Research, 227, 579-589.

6. Galloway, G., Frederick, S., Staggers, F., Gonzales, M., Stalcup, S. \& Smith, D. (1997) Gammahydroxybutyrate: An emerging drug of abuse that causes physical dependency, Addiction, 92, 89-96

7. Kam, P. \& Yoong, F. (1998) Gamma-hydroxybutyric acid: An emerging recreational drug, Anaesthesia, 53, 1195-1198.

8. Bernasconi, R., Mathivet, P., Bischoff, S. \& Marescaux, C. (1999) Gamma-hydroxybutyric acid: An endogenous neuromodulator with abuse potential?, Trends in Pharmacological Science, 20, 135-141.

9. Fattore, L., Martellotta, M., Cossu, G. \& Fratta, W. (2000) Gamma-hydroxybutyric acid: An evaluation of its rewarding properties in rats and mice, Alcohol, 20, 247-256.

10. Snead, O. (1977) Gamma-hydroxybutyrate, Life Sciences, 20, 1935-1944.

11. Gessa, G., Agabio, R., Carai, M., Lobina, C., Pani, M., Reali, R. \& Colombo, G. (2000) Mechanism of anti-alcohol effect of gamma hydroxybutyric acid (GHB), Alcohol, 20, 271276.

12. Colombo, G. \& Gessa, G. L. (2000) Gammahydroxybutyric acid in alcohol preference, dependence and withdrawal, Addiction Biology, 5, 389-403.

13. Colombo, G., Agabio, R., Lobina, C., Reali, R., Fadda, F. \& Gessa, G. (1995) Symmetrical generalisation between the discriminative stimulus effects of gamma-hydroxybutyric acid and ethanol: Occurrence within narrow dose ranges, Physiology and Behavior, 57, 105-111.

14. Vickers, M. (1969) Gammahydroxybutyric acid, International Anaesthesia Clinics, 7, 75-89

15. Mamelak, M. (1989) Gammahydroxybutyrate: An endogenous regulator of energy metabo- lism, Neuroscience and Biobehavior Review, 13, 187-198.

16. Mack, R. (1993) Love potion number 8 1/2, North Carolina Medical Journal, 54, 232-233.

17. Chin, M., Kreutzer, R. \& Dyer, J. (1992) Acute poisoning from gamma-hydroxybutyrate overdose, Annals of Emergency Medicine, 31, 716-722.

18. Scharf, M., Brown, D., Woods, M., Brown, L. \& Hirschowitz, J. (1985) The effects and effectiveness of gamma-hydroxybutyrate in patients with narcolepsy, Journal of Clinical Psychiatry, 46, 222-225.

19. Vickers, M. (1968) Gammahydroxybutyric acid, Proceedings of the Royal Society of Medicine, 61, 821-823.

20. Laborit, H. (1964) Sodium 4 hydroxybutyrate, International Journal of Neuropharmacology, 43, 433-452.

21. Hunter, A., Long, W. \& Ryrie, C. (1971) An evaluation of gamma hydroxybutyric acid in paediatric practice, British Journal of Anaesthesia, 43, 620-627.

22. Takahara, J., Yunoki, S., Yakushiji, W., Yamauchi, J., Yamane, Y. \& Ofuji, T. (1977) Stimulatory effects of gamma-hydroxybutyric acid on growth hormone and prolactin release in humans, Journal of Clinical Endocrinology and Metabolism, 44, 1014-1017.

23. Friedman, J., Westlake, R. \& Furman, M. (1996) "Grievous bodily harm": Gamma hydroxybutyrate abuse leading to the Wernicke-Korsakoff syndrome, Neurology, 46, 469-471.

24. Gallimberti, L., Cibin, M. \& Pagnin, P. (1993) Gamma hydroxybutyric acid for the treatment of opiate withdrawal syndrome, Neuropsychopharmacology, 9, 77-81.

25. Gallimberti, L., Cibin, M. \& Pagnin, P. (Gamma hydroxybutyric acid for the treatment of opiate withdrawal syndrome) Neuropsychopharmacology, 9.

26. Maremmani, I., Lamanna, F. \& Tagliamonte, A. (2001) Long-term therapy using GHB (sodium gamma hydroxybutyrate) for treatment-resistant chronic alcoholics, Journal of Psychoactive Drugs, 33, 135-142.

27. Caputo, F., Addolorato, G., Lorenzini, F., Domenicali, M., Greco, G., Del Re, A., Gasbarrini, G., Stefanini, G. \& Bernardi, M. (2003) Gammahydroxybutyric acid versus naltrexone in maintaining alcohol abstinence: An open randomi- 
sed comparative study, Drug \& Alcohol Dependence, 70, 85-91.

28. United States Department of Health and Human Services (1990) Epidemiologic notes and reports: Multistate outbreak of poisonings associated with illicit use of gamma hydroxybutyrate, Morbidity and Mortality Weekly Report, 39, 861-863.

29. Australian Bureau of Criminal Intelligence (2000) Australian Illicit Drug Report 1998-1999 (Canberra, Australian Bureau of Criminal Intelligence).

30. Breen, C., Topp, L. \& Longo, M. (2002) Adapting the IDRS methodology to monitor trends in party drug markets: Findings of a two year feasibility trial. NDARC Technical Report No. 142 (Sydney, National Drug and Alcohol Research Centre, UNSW).

31. White, B., Degenhardt, L., Breen, C. \& Roxburgh, A. (2003) NSW Party Drug Trends 2002: Findings from the Party Drug Initiative. NDARC Technical Report No. 153 (Sydney, National Drug and Alcohol Research Centre, UNSW).

32. United States Department of Health and Human Services (1997) Gamma hydroxybutyrate use - New York and Texas, 1995-1996, Morbidity and Mortality Weekly Report, 46, 281283.

33. Whitten, L. (2001) Conference highlights increasing GHB abuse, NIDA Notes, 16, 10-11.

34. Thomas, G., Bonner, S. \& Gascoigne, A. (1997) Coma induced by abuse of gamma-hydroxybutyrate (GBH or liquid ecstasy): A case report, British Medical Journal, 314, 35.

35. Weir, E. (2000) Raves: A review of the culture, the drugs and the prevention of harm, Canadian Medical Association Journal, 162, 18431848.

36. Miro, O., Nogue, S., Espinosa, G., To-Figueras, J. \& Sanchez, M. (2002) Trends in illicit drug emergencies: the emerging role of gammahydroxybutyrate, Journal of Toxicology - Clinical Toxicology, 40, 129-35.

37. Ingels, M., Rangan, C., Bellezo, J. \& Clark, R. (2000) Coma and respiratory depression following the ingestion of $\mathrm{GHB}$ and its precursors: Three cases, Journal of Emergency Medicine, 19, 47-50.

38. Miotto, K., Darakjian, J., Basch, J., Murray, S., Zogg, J. \& Rawson, R. (2001) Gammahydroxybutyric acid: Patterns of use, effects and with- drawal, American Journal on Addictions, 10, 232-241.

39. Degenhardt, L., Darke, S. \& Dillon, P. (2002) GHB use among Australians: Characteristics, use patterns, and associated harm, Drug and Alcohol Dependence, 67, 89-94.

40. Topp, L., Hando, J., Dillon, P., Roche, A. \& Solowij, N. (1999) Ecstasy use in Australia: patterns of use and associated harm, Drug and Alcohol Dependence, 55, 105-115.

41. Dyer, J. (1991) Gamma-Hydroxybutyrate: A health food product producing coma and seizure-like activity, American Journal of Emergency Medicine, 9, 321-324.

42. Muller, A. (2003) GHB poisoning: Three recent cases reflect the continuing danger, Journal of Emergency Nursing, 29, 72-74.

43. Sanguineti, V., Angelo, A. \& Frank, M. (1997) GHB: A home brew, American Journal of Drug \& Alcohol Abuse, 23, 637-642

44. Karch, S., Stephens, B. \& Nazareno, G. (2001) GHB: Club drug or confusing artifact?, American Journal of Forensic Medicine and Pathology, 22, 268-269.

45. Li, J., Stokes, S. \& Woeckener, A. (1998) A tale of novel intoxication: Seven cases of gammahydroxybutyrate acid overdose, Annals of Emergency Medicine, 31, 723-728.

46. Eckstein, M., Henderson, S., Delacruz, P. \& Newton, E. (1999) Gamma Hydroxybutyrate (GHB): Report of a mass intoxication and review of the literature, Preshospital Emergency Care, 3, 357-361.

47. Chin, M., Sporer, K., Cullison, B., Dyer, J. \& Wu, T. (1998) Clinical course of gammahydroxybutyrate overdose, Annals of Emergency Medicine, 31, 716-722.

48. Us Drug Enforcement Agency (2001) Drug Intelligence Brief - Club Drugs: An Update (DEA Intelligence Division, Office of Domestic Intelligence, Domestic Strategy Unit).

49. Ferrara, S., Tedeschi, L. \& Frison, G. (1995) Fatality due to gamma-hydroxybutyric acid (GHB) and heroin intoxication, Journal of Forensic Science, 40, 501-504.

50. Marwick, C. (1997) Coma-inducing drug GHB may be reclassified, Journal of the American Medical Association, 277, 1505-1506.

51. Centers for Disease Control (1997) Gamma hydroxybutyrate use - New York and Texas, 
1995-1996, Morbidity and Mortality Weekly Report, 46, 281-283.

52. Degenhardt, L., Darke, S. \& Dillon, P. (2003) The prevalence and correlates of GHB overdose among Australian users, Addiction, 98, 199-204.

53. Mansergh, G., Colfax, G. N., Marks, G., Rader, M., Guzman, R. \& Buchbinder, S. (2001) The Circuit Party Men's Health Survey: findings and implications for gay and bisexual men, American Journal of Public Health., 91, 953-8.

54. Caldicott, D. \& Kuhn, M. (2001) Gammahydroxybutyrate overdose and physostigmine: Teaching new tricks to an old dog?, Annals of Emergency Medicine, 37, 99-102.

55. Li, J., Stokes, S. \& Woeckener, A. (1998) A tale of novel intoxication: A review of the effects of gamma-hydroxybutyric acid with reocmmendations for management, Annals of Emergency Medicine, 31, 729-736.

56. Mcdaniel, C. \& Miotto, K. (2001) Gamma hydroxybutyrate (GHB) and gamma butyrolactone (GBL) withdrawal: Five case studies, Journal of Psychoactive Drugs, 33, 143-149.
57. Craig, K., Gomez, H., Mcmanus, J. \& Bania, T. (2000) Severe gamma-hydroxybutyrate withdrawal: a case report and literature review, Journal of Emergency Medicine, 18, 65-70.

58. Schneir, A., Ly, B. \& Clark, R. (2001) A case of withdrawal from the GHB precursors gammabutyrolactone and 1,4-butanediol, Journal of Emergency Medicine, 21, 31-33.

59. Catalano, M., Glass, J., Catalano, G., Burrows, S., Lynn, W. \& Weitzner, B. (2001) Gamma butyrolactone (GBL) withdrawal syndromes, Psychosomatics, 42, 83-88.

60. Dyer, J., Roth, B. \& Hyme, B. (2001) GammaHydroxybutyrate withdrawal syndrome, Annals of Emergency Medicine, 37, 147-153.

61. Bowles, T., Sommi, R. \& Amiri, M. (2001) Successful management of prolonged gammahydroxybutyrate and alcohol withdrawal, Pharmacotherapy, 21, 254-257.

62. Slaughter, L. (2000) Involvement of drugs involved in sexual assault, Journal of Reproductive Medicine, 45, 425-430. 
